\title{
Assessment of Technologies To Meet a Low Carbon Fuel Standard
}

\section{Supporting Information}

\author{
Sonia Yeh, Ph.D. \\ Research Scientist \\ Institute of Transportation Studies \\ University of California, Davis, One Shields Ave., Davis, CA 95616 \\ (530) 754-9000, Email: slyeh@ucdavis.edu \\ Nicholas Lutsey, Ph.D. \\ Postdoctoral Researcher \\ Institute of Transportation Studies \\ University of California, Davis, One Shields Ave., Davis, CA 95616 \\ Email:nplutsey@ucdavis.edu \\ Nathan Parker \\ Ph.D. Candidate \\ Institute of Transportation Studies \\ University of California, Davis, One Shields Ave., Davis, CA 95616 \\ Email: ncparker@ucdavis.edu
}




\section{Assumptions of Carbon Intensity}

Based on the California Air Resources Board Staff Report's Initial Statement of Reasons (ISOR) (S1), we assume for this analysis that the baseline gasoline and diesel, CARBOB (California Reformulated Gasoline Blendstock for Oxygenate Blending) and ultra-low sulfur diesel (ULSD), have carbon intensity (CI) values of 95.9 and 94.7 $\mathrm{gCO}_{2} \mathrm{e} / \mathrm{MJ}$, respectively, and that the carbon intensities of biofuels are $50.7 \mathrm{gCO}_{2} \mathrm{e} / \mathrm{MJ}$ for advanced corn ethanol; $22.2 \mathrm{gCO}_{2} \mathrm{e} / \mathrm{MJ}$ for cellulosic ethanol from forestry wastes, municipal solid waste (MSW), orchard/vineyard waste, and agricultural residue; and $15 \mathrm{gCO}_{2} \mathrm{e} / \mathrm{MJ}$ for waste-derived biodiesel or renewable diesel including fatty acid to hydrocarbon (FAHC) biodiesel form waste and Fischer-Tropsch (FT) MSW. Greenhouse gas (GHG) emissions of 30, 46, 42, and $18 \mathrm{gCO}_{2} \mathrm{e} / \mathrm{MJ}$ from indirect land use change are added to corn ethanol, Brazilian sugarcane ethanol, soybean biodiesel, and energy crops respectively. A complete list of CI values used in this analysis can be found in the ISOR (S1) Tables IV-20, IV-21, VI-3, and VI-4.

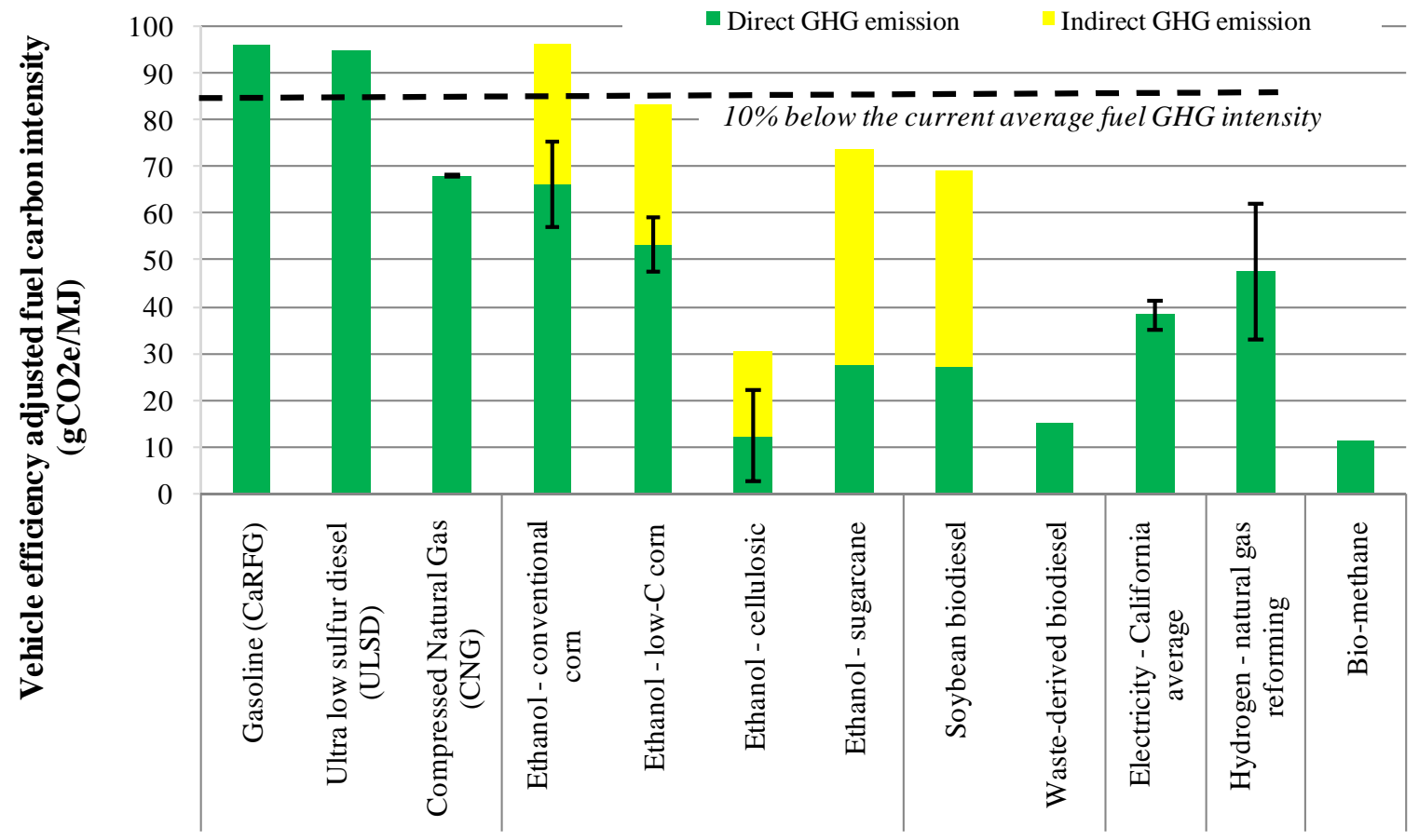

Figure S1. California LCFS greenhouse gas (GHG) intensity ratings $\left(\mathrm{gCO}_{2} \mathrm{e} / \mathrm{MJ}\right)$ for transportation fuels, adjusting for vehicle efficiency factor, the energy economy ratio (EER) (S1). Error bars represent the range of direct lifecycle emissions using different technologies/feedstocks/energy sources (S1). Although no uncertainties are reflected in this graph, the uncertainties of indirect emissions are much larger than the uncertainties of direct emissions.

As shown in Figure S1, a variety of low-GHG fuels from a wide range of feedstocks and production pathways have the potential to substitute for high-GHG gasoline and diesel. The average fuel carbon intensity (AFCI) of a regulated party is calculated as: 


$$
A F C I=\frac{\sum_{i}^{n} E_{i} \times C I_{i}}{\sum_{i}^{n} E_{i} \times E E R_{i}}
$$

Where:

$$
\begin{aligned}
A F C I= & \text { average fuel carbon intensity value of the targeted fuel (gasoline or diesel), in } \mathrm{gCO}_{2} \mathrm{e} / \mathrm{MJ} ; \\
C I_{i}= & \text { carbon intensity value of fuel } i, \text { in } \mathrm{gCO}_{2} \mathrm{e} / \mathrm{MJ} ; \\
E_{i}= & \text { energy of fuel } i \text {, in MJ; } \\
E E R_{i}= & \text { dimensionless Energy Economy Ratio (EER) of fuel } i \text {, which compares the energy } \\
& \text { efficiency of an alternative fuel vehicle to a conventional gasoline or diesel vehicle. }
\end{aligned}
$$

\begin{tabular}{|c|c|c|c|c|c|c|c|}
\hline Reference name & Feedstock & $\begin{array}{l}\text { Conversion } \\
\text { technology }\end{array}$ & Fuel type & 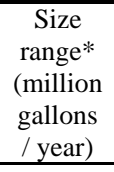 & $\begin{array}{l}\text { Capital cost } \\
\text { (million \$) }\end{array}$ & $\begin{array}{l}\text { Annual O\&M } \\
\text { cost }^{+} \text {(million } \\
\text { \$ / year) }\end{array}$ & $\begin{array}{l}\text { Yield } \\
\text { (gallons } \\
\text { fuel / ton } \\
\text { biomass) }\end{array}$ \\
\hline \multicolumn{8}{|c|}{ Current representative technologies } \\
\hline Grain to ethanol - Dry Mill & Grains/starches & $\begin{array}{l}\text { Enzymatic } \\
\text { fermentation }\end{array}$ & Ethanol & 5 to 100 & $\$ 13$ to $\$ 167$ & $\begin{array}{l}\$ 1.2 \text { to } \\
\$ 16\end{array}$ & 100 \\
\hline Grain to ethanol - Wet Mill & Grains/starches & $\begin{array}{l}\text { Separation and } \\
\text { fermentation }\end{array}$ & Ethanol & $\begin{array}{l}50 \text { to } \\
300\end{array}$ & $\begin{array}{l}\$ 139 \text { to } \\
\$ 408\end{array}$ & $\begin{array}{l}\$ 3.4 \text { to } \\
\$ 21\end{array}$ & 89 \\
\hline $\begin{array}{l}\text { Fatty acid to methyl ester } \\
\text { (FAME) }\end{array}$ & $\begin{array}{l}\text { Seed oil / waste } \\
\text { oils / animal fats }\end{array}$ & Esterfication & Methyl esters & 1 to 80 & $\$ 3$ to $\$ 40$ & $\begin{array}{l}\$ 0.4 \text { to } \\
\$ 17\end{array}$ & 249 to 266 \\
\hline \multicolumn{8}{|c|}{ Additional technologies projected to be in use by 2015 to 2025} \\
\hline $\begin{array}{l}\text { Lignocellulosics to ethanol - } \\
\text { fermentation/hydrolysis (LCE } \\
\text { fermentation/hydrolysis) }\end{array}$ & $\begin{array}{l}\text { Lignocellulosic } \\
\text { biomass }\end{array}$ & $\begin{array}{l}\text { Hydrolysis and } \\
\text { fermentation }\end{array}$ & Ethanol & $\begin{array}{l}20 \text { to } \\
100\end{array}$ & $\$ 61$ to $\$ 305$ & $\$ 0.6$ to $\$ 18$ & 70 to 90 \\
\hline $\begin{array}{l}\text { Lignocellulosics to middle } \\
\text { distillates - Fischer-Tropsch } \\
\text { (LCMD - FT) }\end{array}$ & $\begin{array}{l}\text { Lignocellulosic } \\
\text { biomass }\end{array}$ & $\begin{array}{l}\text { Gasification and } \\
\text { Fischer-Tropsch } \\
\text { synthesis }\end{array}$ & $\begin{array}{l}\text { Middle distillates, } \\
\text { gasoline }\end{array}$ & 7 to 200 & $\begin{array}{l}\$ 250 \text { to } \\
\$ 4,050\end{array}$ & $\begin{array}{l}-\$ 2.3 \text { to } \\
-\$ 157\end{array}$ & 32 to 43 \\
\hline $\begin{array}{l}\text { Lignocellulosics to gasoline - } \\
\text { upgrading/pyrolysis (LCG - } \\
\text { upgrading/pyrolysis) }\end{array}$ & $\begin{array}{l}\text { Lignocellulosic } \\
\text { biomass }\end{array}$ & $\begin{array}{l}\text { Pyrolysis oil } \\
\text { production and } \\
\text { upgrading via } \\
\text { hydrotreatment } \\
\text { /hydrocracking }\end{array}$ & $\begin{array}{l}\text { Bio-oil, diesel, } \\
\text { gasoline }\end{array}$ & $\begin{array}{l}0.65 \text { to } \\
18.6\end{array}$ & $\$ 11$ to $\$ 112$ & $\begin{array}{l}\$ 1.4 \text { to } \\
\$ 19\end{array}$ & 22 to 23 \\
\hline $\begin{array}{l}\text { Fatty Acids to HydroCarbon - } \\
\text { Hydrotreatment (FAHC - } \\
\text { Hydrotreatment) }\end{array}$ & $\begin{array}{l}\text { Seed oil / waste } \\
\text { oils / animal fats }\end{array}$ & $\begin{array}{l}\text { Upgrading via } \\
\text { hydrotreatment }\end{array}$ & Renewable diesel & $\begin{array}{l}15 \text { to } \\
200\end{array}$ & $\$ 10$ to $\$ 37$ & $\begin{array}{l}\$ 2.3 \text { to } \\
\$ 26\end{array}$ & 250 \\
\hline
\end{tabular}

\section{Bioenergy Conversion Technology Characteristics}

A brief summary of biofuel conversion technologies and the assumptions of production costs and yields are given in Table S1. Further detailed information can be found in references (S2-4).

Table S1. Characteristics and assumptions of current and advanced biofuel conversion technologies. Source: (S24). 


\section{Biofuel Supply Curves in California and in the Western States}

Quantitative assessment of resources and costs of delivery associated with each individual and applicable biomass resource, combined with Geographic Information System (GIS) modeling in conjunction with an infrastructure system cost optimization model, are used to develop biofuel supply curves using biomass feedstocks throughout California and the western US. The estimated production cost excludes local delivery costs from distribution terminals to refueling stations, market costs, and taxes. The methodologies, assumptions, and additional uncertainty analysis are described in detail in a series of reports that can be found at http://www.westgov.org/wga/initiatives/transfuels/index.html. The estimated biofuel resource supply curves from various feedstocks and processes in California are shown in Figure S2, which estimates the production supply curves of gasoline substitutes that involve corn-derived ethanol as well as lignocellulosic ethanol (LCE) from forest, orchard waste, agricultural residue, and municipal solid waste (MSW). Similarly, production supply curves are constructed for diesel substitutes such as fatty acid to hydrocarbon (FAHC) conversion of tallow and grease and for Fischer-Tropsch (FT) production of biomass-based diesel from municipal solid waste. The figure shows that the largest resource estimated to be available from within California is MSW, which includes mixed paper, wood wastes, and yard wastes. Corn is not found to be a significant source of biofuel resource in California, and energy crops are not estimated to be economically attractive within the state.

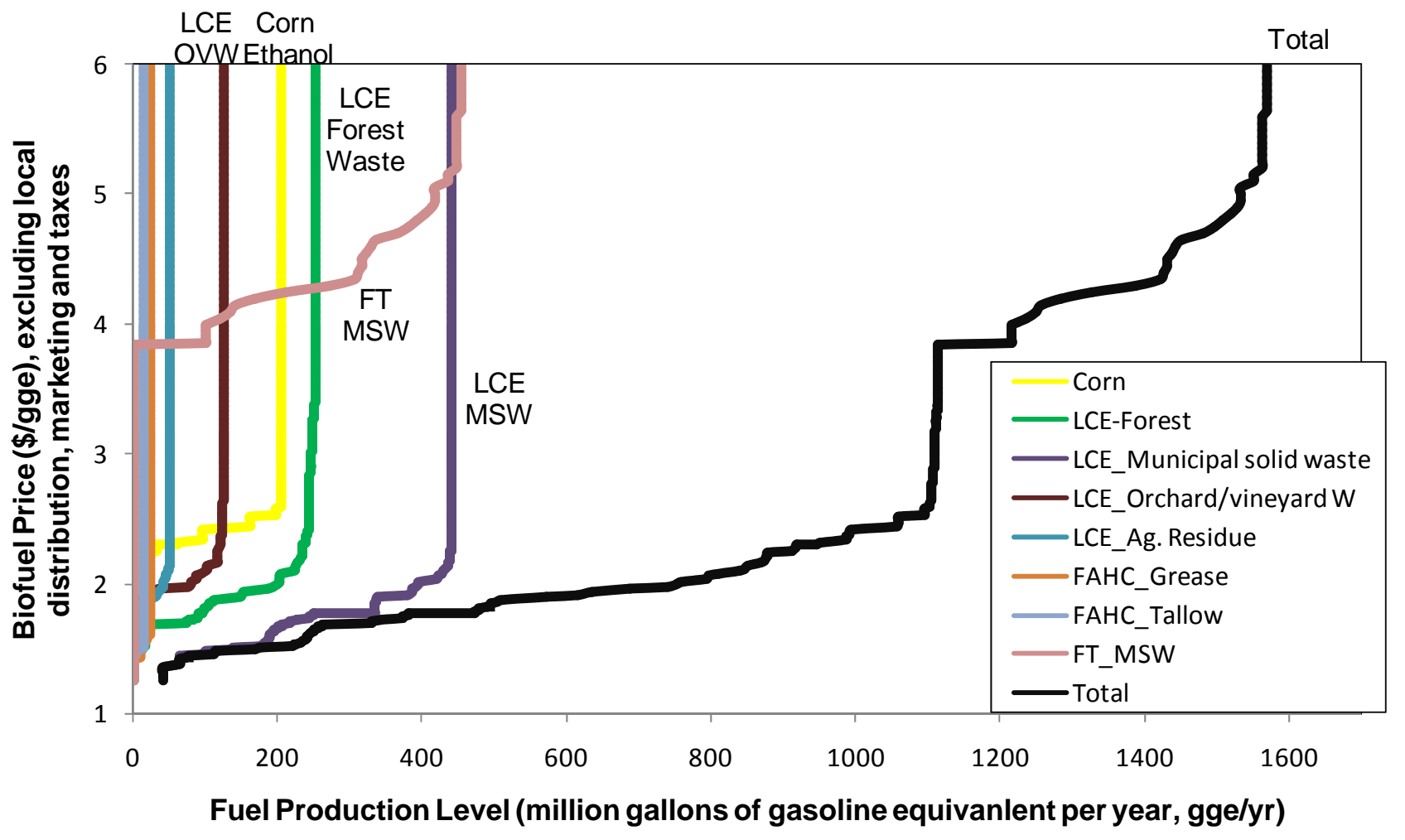

Figure S2. Biofuel production supply curves in California by biomass and technology type. The three lines on the far left are FAHC-tallow, FAHC-grease, and LCE-agricultural residue (from left to right). Abbreviations: LCE=lignocellulosic ethanol; FT=Fischer-Tropsch; MSW=municipal solid waste; OVW=orchard/vine waste. 
The total resource supply curves for the western states are summarized in Figure S3 by biomass feedstock and in Figure S4 by biofuel type.

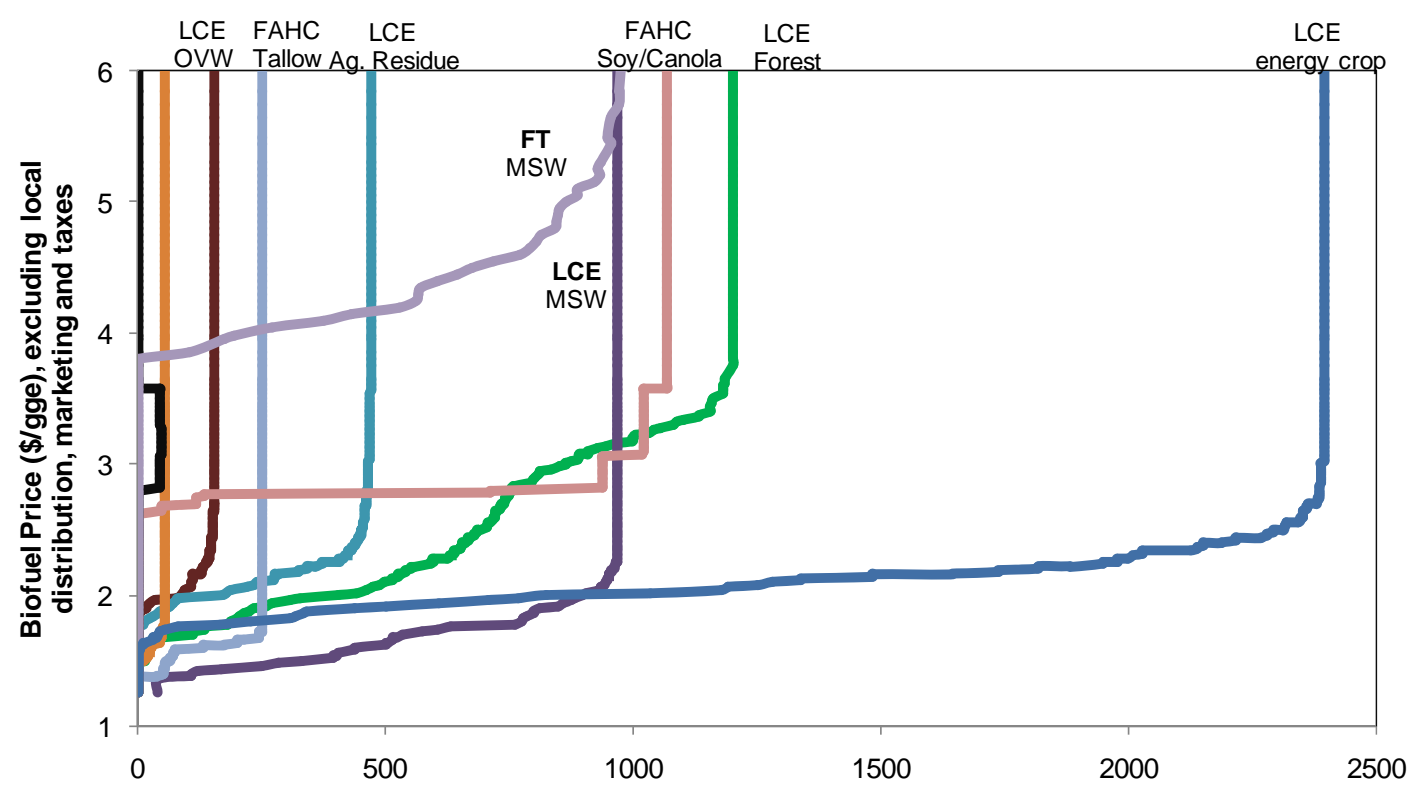

Fuel Production Level (million gallons of gasoline equivanlent per year, gge/yr)

Figure S3. Biofuel production supply curves in the western states by biomass feedstock. Corn ethanol is off-scale and is shown in Figure S4. The two lines on the far left are FAME-soy/canola and FAHC-grease (from left to right).

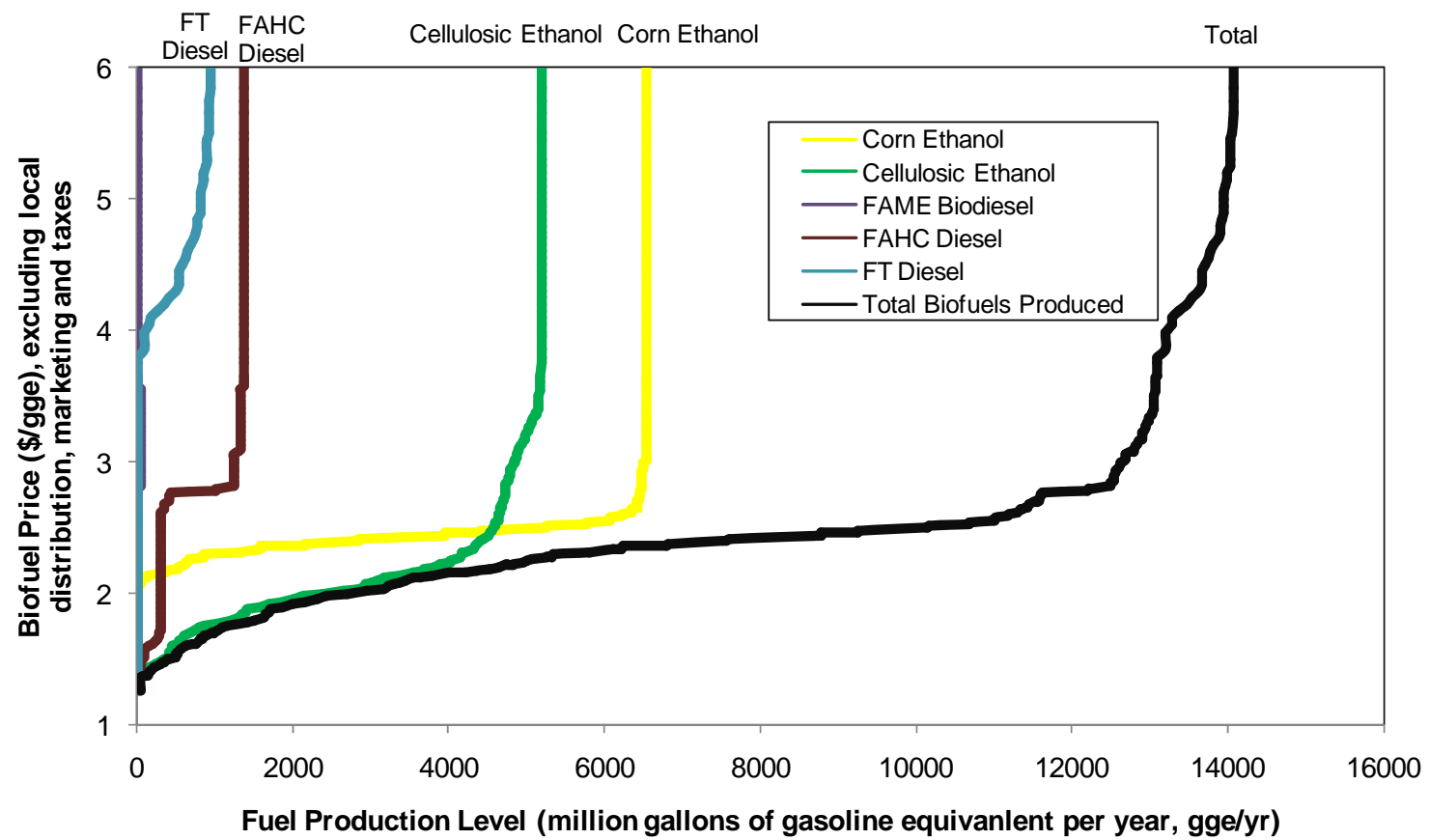

Figure S4. Biofuel production supply curves in the western states by biofuel type. Abbreviations: FAHC=fatty acid to hydrocarbon; FT=Fischer-Tropsch. 


\section{Fuel Providers’ Compliance Cost-Effectiveness Curves}

Fuel providers' compliance cost-effectiveness curves by feedstock, as calculated based on Equation 2 in the main text, are shown in Figure S5 for biofuels from California and Figure S6 from the western states.

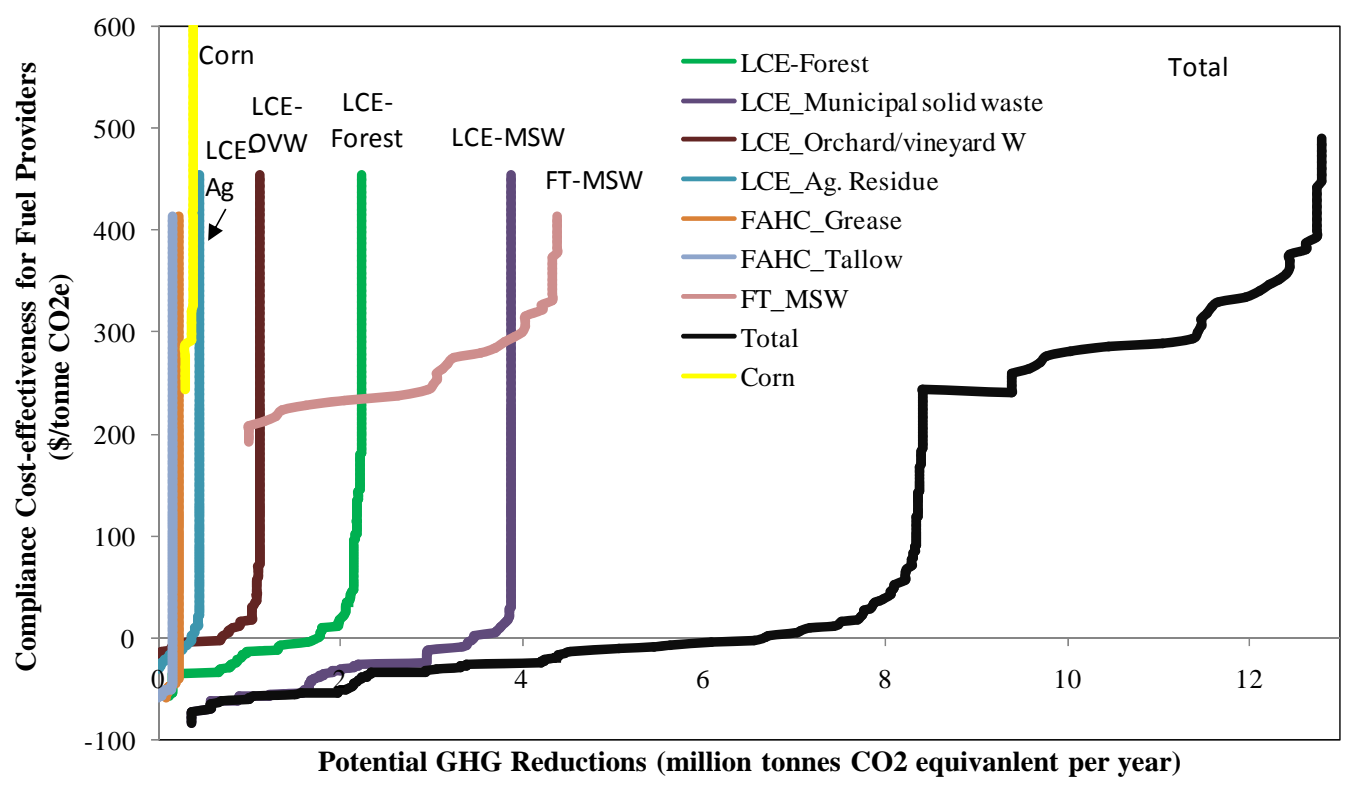

Figure S5. Fuel providers' biofuel GHG compliance cost-effectiveness curves from California by feedstock at gasoline fuel cost of \$2.0/gge (production cost, excluding local delivery, marketing, and taxes). Abbreviations: LCE=lignocellulosic ethanol; FAME=fatty acid to methyl esters; FAHC=fatty acid to hydrocarbon; FT=FischerTropsch; MSW=municipal solid waste; OVW=orchard/vine waste; Ag=agricultural residue.

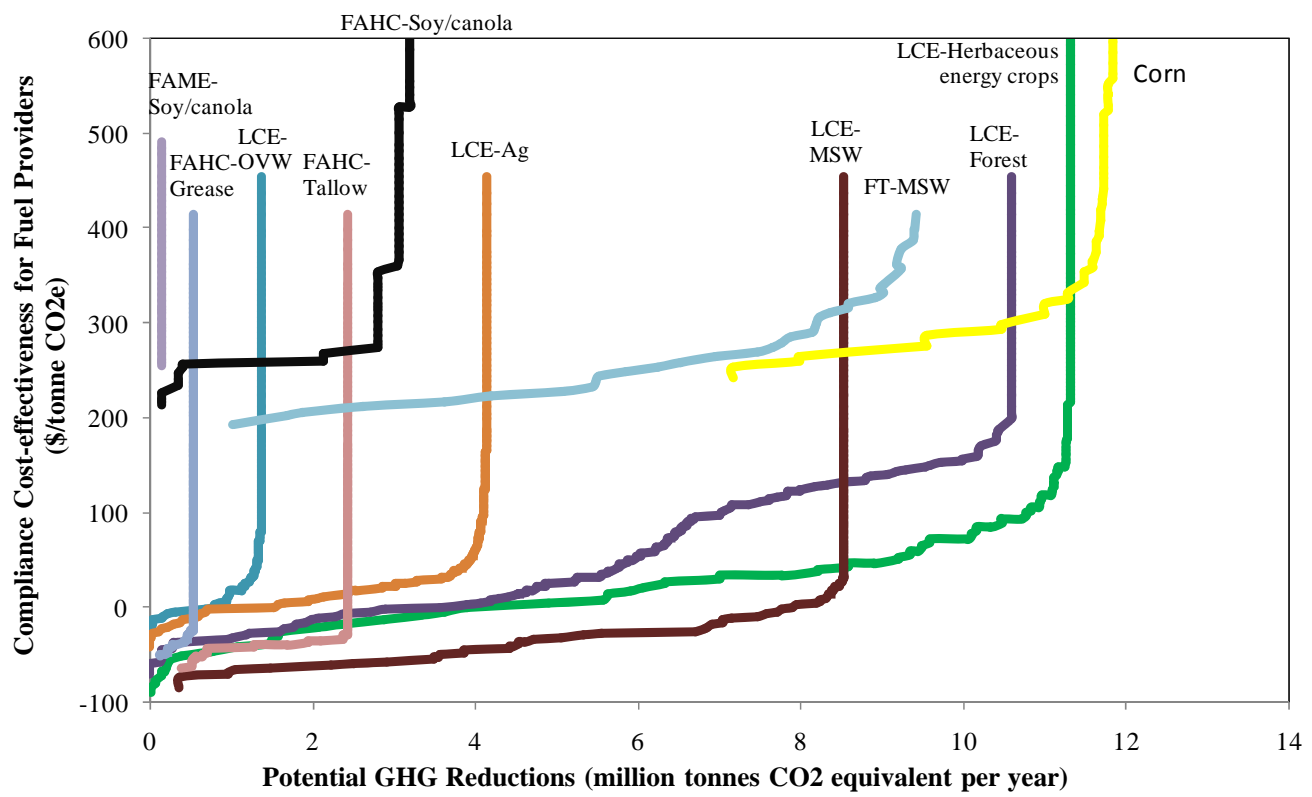

Figure S6. Fuel providers' biofuel GHG compliance cost-effectiveness curves from western states by feedstock at a gasoline fuel cost of \$2.0/gge (production cost, excluding local delivery, marketing, and taxes).

VISION-CI model 
The original VISION model was developed by Argonne National Laboratory (S5) and subsequently modified for California-specific data to become our VISION-CA model (S6). VISION-CI (CI standards for carbon intensity) used for this analysis is an updated version of the earlier VISION-CA model. The VISION models are vehiclefleet turnover models that track vehicles, fuel penetration, and fleet turnover by its vintage years and allow for user-defined changes in fuel use. The rate of substitution of an existing vehicle fleet by more fuel-efficient vehicles or flex-fuel vehicles is limited by the rate of vehicle turnover and the maximum rate of introduction of new technology, which is assumed to have an S-shaped logistical function adoption curve. The model documentation can be found at the VISION model website (S5).

The reference case incorporates California’s AB1493 (Pavley), which sets vehicle emission performance standard that requires a 30\% reduction in GHG emissions rate from new light-duty vehicles by 2016 (S7). The E10 requirement for $10 \%$ ethanol in gasoline after 2010 is also assumed in the base case (S8). When E85 flex-fuel vehicles are introduced to utilize lower-GHG biofuels, we assume that their E85 mileage share (the percentage of time the vehicle runs on E85) will be $50 \%$ in 2020 in the LCFS compliance scenario. Ethanol, which can be derived from various agricultural and waste feedstocks, can be mixed with gasoline up to 10\% by volume (E10) without any vehicle modifications, and up to $85 \%$ by volume (E85) in flex-fuel vehicles. Traditional biodiesel (fatty acid methyl ester [FAME]) from lipid feedstocks can be blended into diesel fuel up to 20\% (B20) without major vehicle modifications. Advanced biomass-based diesels produced through the Fischer-Tropsch or hydrotreatment processes are expected to blend into diesel fuel without restriction. Applications for electrification span light-duty, heavy-duty, and non-road vehicles and generally include electric battery storage, electric motors, and charging equipment to allow vehicles to utilize grid-connection electricity to supplant conventional gasoline and diesel energy sources.

Total fuel use and vehicle penetration for light-duty (LDV) and heavy-duty (HDV) ${ }^{1}$ vehicles are shown in Table S2 for the reference case and Table S3 for the portfolio scenario.

\footnotetext{
${ }^{1}$ Gross vehicle weight $>10,000$ lbs.
} 
Table S2. Transportation fuel use and vehicle penetration in the reference case.

\begin{tabular}{|c|c|c|c|c|c|c|c|c|c|c|c|}
\hline \multicolumn{12}{|c|}{ LDV transportation fuel use (Quads/yr) ${ }^{2}$} \\
\hline & 2010 & 2011 & 2012 & 2013 & 2014 & 2015 & 2016 & 2017 & 2018 & 2019 & 2020 \\
\hline Gasoline & 1.99 & 2.02 & 2.04 & 2.06 & 2.06 & 2.05 & 2.03 & 2.03 & 2.00 & 1.97 & 1.89 \\
\hline Ethanol & 0.14 & 0.15 & 0.15 & 0.15 & 0.15 & 0.15 & 0.15 & 0.15 & 0.15 & 0.15 & 0.15 \\
\hline Diesel & 0.03 & 0.03 & 0.03 & 0.03 & 0.03 & 0.03 & 0.04 & 0.04 & 0.04 & 0.04 & 0.05 \\
\hline Electricity & 0.00 & 0.00 & 0.00 & 0.00 & 0.00 & 0.00 & 0.00 & 0.00 & 0.00 & 0.00 & 0.00 \\
\hline Compressed natural gas (CNG) & 0.00 & 0.00 & 0.00 & 0.00 & 0.00 & 0.00 & 0.00 & 0.00 & 0.00 & 0.00 & 0.00 \\
\hline Hydrogen & 0.00 & 0.00 & 0.00 & 0.00 & 0.00 & 0.00 & 0.00 & 0.00 & 0.00 & 0.00 & 0.00 \\
\hline Total & 2.16 & 2.20 & 2.22 & 2.25 & 2.25 & 2.24 & 2.22 & 2.22 & 2.20 & 2.16 & 2.09 \\
\hline \multicolumn{12}{|c|}{ LDV fleet market share (\%), based on VISION-CI model } \\
\hline & 2010 & 2011 & 2012 & 2013 & 2014 & 2015 & 2016 & 2017 & 2018 & 2019 & 2020 \\
\hline $\begin{array}{l}\text { Conventional gasoline and } \\
\text { gasoline hybrid vehicle }\end{array}$ & $95.2 \%$ & $94.3 \%$ & $93.3 \%$ & $92.2 \%$ & $91.0 \%$ & $89.6 \%$ & $88.1 \%$ & $86.5 \%$ & $84.9 \%$ & $83.2 \%$ & $81.5 \%$ \\
\hline Battery electric vehicle & $0.0 \%$ & $0.0 \%$ & $0.0 \%$ & $0.0 \%$ & $0.0 \%$ & $0.0 \%$ & $0.0 \%$ & $0.0 \%$ & $0.0 \%$ & $0.0 \%$ & $0.0 \%$ \\
\hline E85 flex-fuel vehicle & $3.1 \%$ & $3.9 \%$ & $4.8 \%$ & $5.9 \%$ & $7.1 \%$ & $8.3 \%$ & $9.7 \%$ & $11.0 \%$ & $12.5 \%$ & $13.9 \%$ & $15.3 \%$ \\
\hline Diesel vehicle & $1.6 \%$ & $1.7 \%$ & $1.7 \%$ & $1.8 \%$ & $1.9 \%$ & $2.0 \%$ & $2.1 \%$ & $2.2 \%$ & $2.4 \%$ & $2.6 \%$ & $2.8 \%$ \\
\hline Compressed natural gas vehicle & $0.1 \%$ & $0.1 \%$ & $0.1 \%$ & $0.1 \%$ & $0.1 \%$ & $0.1 \%$ & $0.1 \%$ & $0.1 \%$ & $0.1 \%$ & $0.1 \%$ & $0.1 \%$ \\
\hline Plug-in hybrid vehicle & $0.0 \%$ & $0.0 \%$ & $0.0 \%$ & $0.0 \%$ & $0.0 \%$ & $0.0 \%$ & $0.0 \%$ & $0.0 \%$ & $0.1 \%$ & $0.1 \%$ & $0.1 \%$ \\
\hline Plug-in hybrid diesel vehicle & $0.0 \%$ & $0.0 \%$ & $0.0 \%$ & $0.0 \%$ & $0.0 \%$ & $0.0 \%$ & $0.0 \%$ & $0.0 \%$ & $0.0 \%$ & $0.0 \%$ & $0.0 \%$ \\
\hline Hydrogen fuel cell vehicle & $0.0 \%$ & $0.0 \%$ & $0.0 \%$ & $0.0 \%$ & $0.0 \%$ & $0.0 \%$ & $0.0 \%$ & $0.0 \%$ & $0.0 \%$ & $0.0 \%$ & $0.0 \%$ \\
\hline Sum & $100 \%$ & $100 \%$ & $100 \%$ & $100 \%$ & $100 \%$ & $100 \%$ & $100 \%$ & $100 \%$ & $100 \%$ & $100 \%$ & $100 \%$ \\
\hline \multicolumn{12}{|c|}{ HDV transportation fuel use (Quads/yr) } \\
\hline & 2010 & 2011 & 2012 & 2013 & 2014 & 2015 & 2016 & 2017 & 2018 & 2019 & 2020 \\
\hline Gasoline & 0.031 & 0.031 & 0.032 & 0.032 & 0.032 & 0.033 & 0.033 & 0.034 & 0.034 & 0.035 & 0.035 \\
\hline Ethanol & 0.002 & 0.002 & 0.002 & 0.002 & 0.002 & 0.002 & 0.002 & 0.002 & 0.002 & 0.003 & 0.003 \\
\hline Diesel & 0.432 & 0.443 & 0.455 & 0.468 & 0.483 & 0.499 & 0.512 & 0.525 & 0.537 & 0.549 & 0.559 \\
\hline liquefied petroleum gas (LPG) & 0.008 & 0.008 & 0.008 & 0.009 & 0.009 & 0.009 & 0.010 & 0.010 & 0.010 & 0.010 & 0.010 \\
\hline CNG & 0.013 & 0.013 & 0.014 & 0.014 & 0.014 & 0.015 & 0.015 & 0.015 & 0.016 & 0.016 & 0.016 \\
\hline Total & 0.49 & 0.50 & 0.51 & 0.53 & 0.54 & 0.56 & 0.57 & 0.59 & 0.60 & 0.61 & 0.62 \\
\hline \multicolumn{12}{|c|}{ HDV fleet market share (\%), based on VISION-CI model } \\
\hline & 2010 & 2011 & 2012 & 2013 & 2014 & 2015 & 2016 & 2017 & 2018 & 2019 & 2020 \\
\hline Gasoline trucks & $17.3 \%$ & $16.8 \%$ & $16.7 \%$ & $16.5 \%$ & $16.7 \%$ & $16.8 \%$ & $16.5 \%$ & $16.4 \%$ & $16.6 \%$ & $16.9 \%$ & $16.8 \%$ \\
\hline Diesel trucks & $51.2 \%$ & $52.1 \%$ & $52.8 \%$ & $53.6 \%$ & $54.4 \%$ & $55.0 \%$ & $54.5 \%$ & $54.5 \%$ & $54.6 \%$ & $54.5 \%$ & $54.5 \%$ \\
\hline LPG trucks & $11.3 \%$ & $11.2 \%$ & $11.0 \%$ & $10.8 \%$ & $10.4 \%$ & $10.2 \%$ & $10.4 \%$ & $10.5 \%$ & $10.4 \%$ & $10.3 \%$ & $10.4 \%$ \\
\hline CNG trucks & $20.1 \%$ & $19.9 \%$ & $19.5 \%$ & $19.1 \%$ & $18.5 \%$ & $18.1 \%$ & $18.5 \%$ & $18.6 \%$ & $18.4 \%$ & $18.3 \%$ & $18.3 \%$ \\
\hline \multicolumn{12}{|c|}{ Total on-road transportation fuel use (billion gasoline gallon equivalent per year, BGGE/yr) } \\
\hline & 2010 & 2011 & 2012 & 2013 & 2014 & 2015 & 2016 & 2017 & 2018 & 2019 & 2020 \\
\hline CARBOB & 17.83 & 18.10 & 18.30 & 18.49 & 18.46 & 18.37 & 18.21 & 18.21 & 17.97 & 17.65 & 16.96 \\
\hline Ethanol & 1.30 & 1.32 & 1.34 & 1.36 & 1.36 & 1.37 & 1.37 & 1.36 & 1.36 & 1.35 & 1.35 \\
\hline CA ULSD & 4.06 & 4.16 & 4.27 & 4.40 & 4.54 & 4.69 & 4.82 & 4.95 & 5.07 & 5.20 & 5.32 \\
\hline Biodiesel & 0.00 & 0.00 & 0.00 & 0.00 & 0.00 & 0.00 & 0.00 & 0.00 & 0.00 & 0.00 & 0.00 \\
\hline Electricity & 0.00 & 0.00 & 0.00 & 0.00 & 0.00 & 0.00 & 0.00 & 0.00 & 0.00 & 0.01 & 0.01 \\
\hline LPG & 0.07 & 0.07 & 0.07 & 0.08 & 0.08 & 0.08 & 0.08 & 0.09 & 0.09 & 0.09 & 0.09 \\
\hline CNG & 0.13 & 0.14 & 0.14 & 0.14 & 0.15 & 0.15 & 0.15 & 0.16 & 0.16 & 0.16 & 0.17 \\
\hline Hydrogen & 0.00 & 0.00 & 0.00 & 0.00 & 0.00 & 0.00 & 0.00 & 0.00 & 0.00 & 0.00 & 0.00 \\
\hline Total & 23.39 & 23.79 & 24.13 & 24.46 & 24.59 & 24.66 & 24.63 & 24.77 & 24.66 & 24.46 & 23.90 \\
\hline
\end{tabular}

\footnotetext{
${ }^{2} 1.0$ Quad = One quadrillion Btu $\left(10^{15} \mathrm{Btu}\right)=1.055$ exajoules $(\mathrm{EJ})$, or approximately 172 million barrels of oil equivalent.
} 
Table S3. Transportation fuel use and vehicle penetration in the portfolio scenario.

\begin{tabular}{|c|c|c|c|c|c|c|c|c|c|c|c|}
\hline \multicolumn{12}{|c|}{ LDV transportation fuel use (Quads/yr) } \\
\hline & 2010 & 2011 & 2012 & 2013 & 2014 & 2015 & 2016 & 2017 & 2018 & 2019 & 2020 \\
\hline Gasoline & 1.98 & 2.01 & 2.02 & 2.02 & 2.00 & 1.96 & 1.91 & 1.91 & 1.85 & 1.79 & 1.65 \\
\hline Ethanol & 0.14 & 0.15 & 0.16 & 0.16 & 0.17 & 0.18 & 0.20 & 0.21 & 0.23 & 0.25 & 0.28 \\
\hline Diesel & 0.03 & 0.03 & 0.03 & 0.03 & 0.03 & 0.03 & 0.04 & 0.04 & 0.04 & 0.04 & 0.05 \\
\hline Electricity & 0.00 & 0.00 & 0.00 & 0.00 & 0.00 & 0.00 & 0.00 & 0.00 & 0.00 & 0.01 & 0.01 \\
\hline CNG & 0.00 & 0.00 & 0.00 & 0.00 & 0.00 & 0.00 & 0.01 & 0.01 & 0.01 & 0.01 & 0.01 \\
\hline Hydrogen & 0.00 & 0.00 & 0.00 & 0.00 & 0.00 & 0.00 & 0.00 & 0.00 & 0.00 & 0.00 & 0.00 \\
\hline Total & 2.16 & 2.19 & 2.21 & 2.22 & 2.21 & 2.18 & 2.15 & 2.18 & 2.14 & 2.10 & 2.00 \\
\hline \multicolumn{12}{|c|}{ LDV fleet market share (\%), based on VISION-CI model } \\
\hline & 2010 & 2011 & 2012 & 2013 & 2014 & 2015 & 2016 & 2017 & 2018 & 2019 & 2020 \\
\hline $\begin{array}{l}\text { Conventional gasoline and } \\
\text { gasoline hybrid vehicle }\end{array}$ & $94.8 \%$ & $93.7 \%$ & $92.4 \%$ & $90.8 \%$ & $88.9 \%$ & $86.7 \%$ & $84.4 \%$ & $81.9 \%$ & $79.3 \%$ & $76.6 \%$ & $73.8 \%$ \\
\hline Battery electric vehicle & $0.0 \%$ & $0.0 \%$ & $0.0 \%$ & $0.0 \%$ & $0.0 \%$ & $0.0 \%$ & $0.1 \%$ & $0.1 \%$ & $0.1 \%$ & $0.1 \%$ & $0.2 \%$ \\
\hline E85 flex-fuel vehicle ${ }^{*}$ & $3.4 \%$ & $4.4 \%$ & $5.6 \%$ & $7.0 \%$ & $8.6 \%$ & $10.5 \%$ & $12.4 \%$ & $14.4 \%$ & $16.5 \%$ & $18.4 \%$ & $20.3 \%$ \\
\hline Diesel vehicle & $1.6 \%$ & $1.7 \%$ & $1.7 \%$ & $1.8 \%$ & $1.9 \%$ & $2.0 \%$ & $2.1 \%$ & $2.2 \%$ & $2.4 \%$ & $2.6 \%$ & $2.8 \%$ \\
\hline Compressed natural gas vehicle & $0.1 \%$ & $0.1 \%$ & $0.1 \%$ & $0.1 \%$ & $0.2 \%$ & $0.2 \%$ & $0.2 \%$ & $0.3 \%$ & $0.3 \%$ & $0.4 \%$ & $0.5 \%$ \\
\hline Plug-in hybrid vehicle & $0.0 \%$ & $0.1 \%$ & $0.2 \%$ & $0.2 \%$ & $0.3 \%$ & $0.5 \%$ & $0.7 \%$ & $0.9 \%$ & $1.2 \%$ & $1.6 \%$ & $2.1 \%$ \\
\hline Plug-in hybrid diesel vehicle & $0.0 \%$ & $0.0 \%$ & $0.0 \%$ & $0.0 \%$ & $0.0 \%$ & $0.0 \%$ & $0.0 \%$ & $0.0 \%$ & $0.0 \%$ & $0.0 \%$ & $0.0 \%$ \\
\hline Hydrogen fuel cell vehicle & $0.0 \%$ & $0.0 \%$ & $0.0 \%$ & $0.0 \%$ & $0.0 \%$ & $0.0 \%$ & $0.0 \%$ & $0.0 \%$ & $0.0 \%$ & $0.0 \%$ & $0.0 \%$ \\
\hline Sum & $100 \%$ & $100 \%$ & $100 \%$ & $100 \%$ & $100 \%$ & $100 \%$ & $100 \%$ & $100 \%$ & $100 \%$ & $100 \%$ & $100 \%$ \\
\hline \multicolumn{12}{|c|}{ HDV transportation fuel use (Quads/yr) } \\
\hline & 2010 & 2011 & 2012 & 2013 & 2014 & 2015 & 2016 & 2017 & 2018 & 2019 & 2020 \\
\hline Gasoline & 0.031 & 0.031 & 0.032 & 0.032 & 0.032 & 0.033 & 0.033 & 0.034 & 0.034 & 0.035 & 0.035 \\
\hline Ethanol & 0.002 & 0.002 & 0.002 & 0.002 & 0.002 & 0.002 & 0.002 & 0.002 & 0.002 & 0.003 & 0.003 \\
\hline Diesel & 0.432 & 0.443 & 0.455 & 0.468 & 0.483 & 0.499 & 0.512 & 0.525 & 0.537 & 0.549 & 0.559 \\
\hline LPG & 0.008 & 0.008 & 0.009 & 0.009 & 0.009 & 0.010 & 0.010 & 0.010 & 0.010 & 0.011 & 0.011 \\
\hline CNG & 0.014 & 0.014 & 0.015 & 0.016 & 0.016 & 0.017 & 0.018 & 0.019 & 0.019 & 0.020 & 0.021 \\
\hline Total & 0.49 & 0.50 & 0.51 & 0.53 & 0.54 & 0.56 & 0.58 & 0.59 & 0.60 & 0.62 & 0.63 \\
\hline \multicolumn{12}{|c|}{ HDV fleet market share (\%), based on VISION-CI model } \\
\hline & 2010 & 2011 & 2012 & 2013 & 2014 & 2015 & 2016 & 2017 & 2018 & 2019 & 2020 \\
\hline Gasoline & $16.7 \%$ & $16.1 \%$ & $15.9 \%$ & $15.7 \%$ & $15.8 \%$ & $15.8 \%$ & $15.5 \%$ & $15.3 \%$ & $15.4 \%$ & $15.6 \%$ & $15.4 \%$ \\
\hline Diesel & $49.4 \%$ & $49.9 \%$ & $50.4 \%$ & $50.8 \%$ & $51.4 \%$ & $51.7 \%$ & $50.9 \%$ & $50.6 \%$ & $50.5 \%$ & $50.2 \%$ & $49.9 \%$ \\
\hline LPG & $10.9 \%$ & $10.8 \%$ & $10.5 \%$ & $10.2 \%$ & $9.8 \%$ & $9.6 \%$ & $9.7 \%$ & $9.7 \%$ & $9.6 \%$ & $9.5 \%$ & $9.5 \%$ \\
\hline CNG & $23.0 \%$ & $23.3 \%$ & $23.3 \%$ & $23.3 \%$ & $23.0 \%$ & $22.9 \%$ & $23.9 \%$ & $24.4 \%$ & $24.5 \%$ & $24.7 \%$ & $25.2 \%$ \\
\hline \multicolumn{12}{|c|}{ Total on-road transportation fuel use (BGGE/yr) } \\
\hline & 2010 & 2011 & 2012 & 2013 & 2014 & 2015 & 2016 & 2017 & 2018 & 2019 & 2020 \\
\hline CARBOB & 17.80 & 18.00 & 18.09 & 18.12 & 17.89 & 17.58 & 17.16 & 17.17 & 16.66 & 16.09 & 14.87 \\
\hline Ethanol & 1.30 & 1.34 & 1.40 & 1.47 & 1.55 & 1.65 & 1.77 & 1.92 & 2.08 & 2.27 & 2.48 \\
\hline CA ULSD & 4.06 & 4.11 & 4.16 & 4.22 & 4.29 & 4.37 & 4.43 & 4.48 & 4.52 & 4.56 & 4.60 \\
\hline Biodiesel & 0.00 & 0.06 & 0.12 & 0.18 & 0.25 & 0.32 & 0.39 & 0.47 & 0.55 & 0.64 & 0.73 \\
\hline Electricity & 0.00 & 0.01 & 0.01 & 0.02 & 0.02 & 0.03 & 0.04 & 0.05 & 0.07 & 0.09 & 0.11 \\
\hline LPG & 0.07 & 0.07 & 0.08 & 0.08 & 0.08 & 0.09 & 0.09 & 0.09 & 0.09 & 0.09 & 0.10 \\
\hline CNG & 0.14 & 0.15 & 0.00 & 0.00 & 0.00 & 0.19 & 0.16 & 0.16 & 0.17 & 0.18 & 0.29 \\
\hline Hydrogen & 0.00 & 0.00 & 0.00 & 0.00 & 0.00 & 0.00 & 0.00 & 0.00 & 0.00 & 0.00 & 0.00 \\
\hline Total & 23.37 & 23.74 & 23.86 & 24.09 & 24.08 & 24.23 & 24.04 & 24.34 & 24.14 & 23.92 & 23.18 \\
\hline
\end{tabular}

"Several major automakers have plans to increase their E85 vehicle sales to 50\% of their new vehicle sales by model year 2014. See (S9-11). 
Based on Equation S1, the trajectories of AFCI-gasoline (the average carbon intensity values of gasoline and gasoline-substitutes) and AFCI-diesel (the average carbon intensity values of diesel and diesel-substitutes) for the reference case and the portfolio scenario are shown in Figure S7.

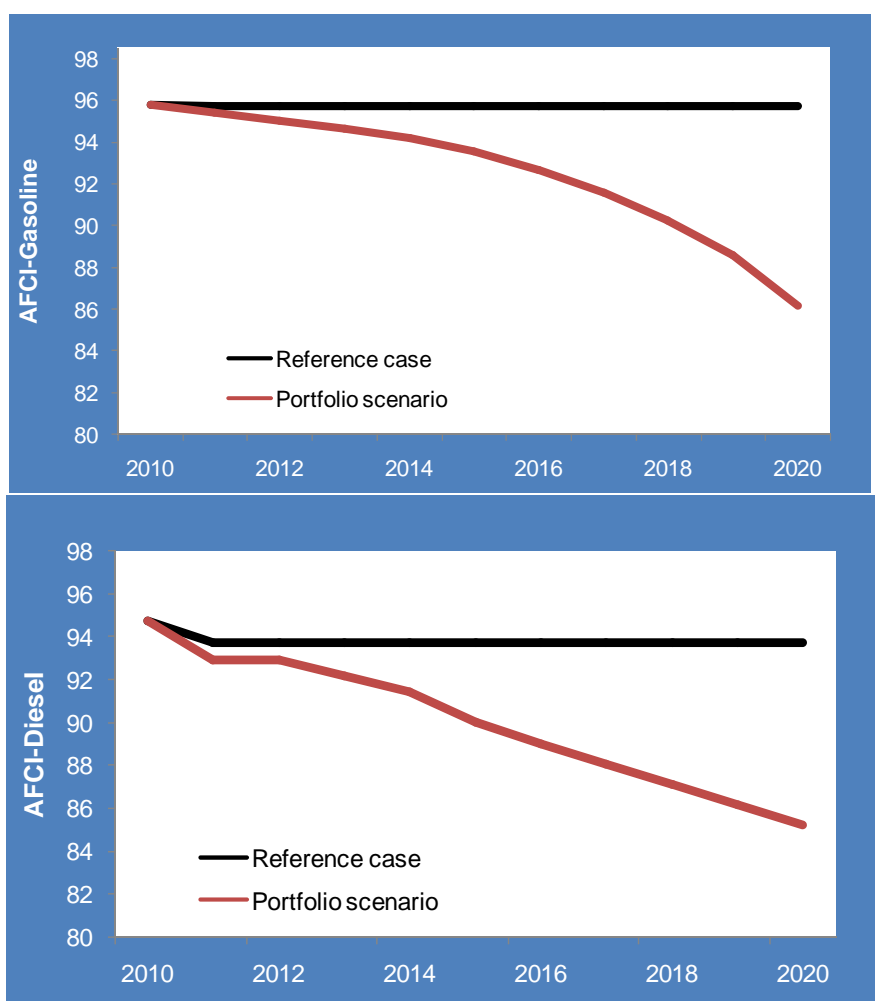

Figure S7. Trajectories of AFCI-gasoline and AFCI-diesel for the reference case, and the portfolio scenario that meets the $10 \%$ AFCI reduction goals. 


\section{National LCFS Analysis}

Tables S4 and S5 show the first-order estimates of fuel use and $\mathrm{CO}_{2} \mathrm{e}$ emissions, and the amount of emission reduction required to achieve a 10\% AFCI reduction target if the US adopts a California-like LCFS. Table S4 is based on the Annual Energy Outlook 2009 reference case (S12), and Table S5 assumes that the RFS target mandated in the Energy Independence and Security Act (EISA) will be fully met. Instead of meeting separate AFCI-gasoline and AFCI-diesel targets, the following calculations are based on the compliance of one AFCI target since over-compliance credits can be traded between the two targets. Table S5 shows that the implementation of the federal biofuel target mandated in the Energy Independence and Security Act (S13) would reduce the average transport-fuel carbon intensity by 3.3\% by 2020 and $4.5 \%$ by 2022 .

Table S4. Estimates of fuel use and $\mathrm{CO}_{2} \mathrm{e}$ emission reduction required to achieve a $10 \%$ AFCI reduction target based on the Annual Energy Outlook 2009 projections (S12).

\begin{tabular}{|c|c|c|c|c|c|c|c|c|c|c|c|c|c|c|}
\hline \multirow[b]{2}{*}{ Fuel type } & \multicolumn{14}{|c|}{ Transportation fuel use (BGGE/yr) } \\
\hline & 2010 & 2011 & 2012 & 2013 & 2014 & 2015 & 2016 & 2017 & 2018 & 2019 & 2020 & $\begin{array}{l}\text { CI } \\
(2010)\end{array}$ & $\begin{array}{l}\text { CI } \\
(2020)\end{array}$ & EER \\
\hline Gasoline & 140.5 & 143.9 & 143.6 & 142.6 & 141.3 & 139.4 & 137.3 & 135.6 & 133.7 & 132.5 & 131.0 & 95.9 & 95.9 & 1 \\
\hline Ethanol & 10.5 & 10.7 & 10.7 & 10.6 & 10.5 & 10.8 & 11.2 & 11.5 & 12.1 & 12.9 & 13.9 & 99.4 & $64.0^{*}$ & 1 \\
\hline Diesel & 62.1 & 63.6 & 65.3 & 66.5 & 66.8 & 67.0 & 67.5 & 68.2 & 69.0 & 69.6 & 70.4 & 94.7 & 94.7 & 1 \\
\hline Biodiesel & 0.9 & 1.0 & 1.3 & 1.6 & 1.8 & 2.1 & 2.3 & 2.6 & 2.9 & 3.1 & 3.4 & 48.0 & 48.0 & 1 \\
\hline Electricity & 0.2 & 0.2 & 0.2 & 0.2 & 0.2 & 0.2 & 0.2 & 0.2 & 0.3 & 0.3 & 0.3 & 164.4 & 164.4 & 3 \\
\hline LPG & 0.1 & 0.1 & 0.1 & 0.1 & 0.1 & 0.1 & 0.1 & 0.1 & 0.1 & 0.1 & 0.1 & 77.3 & 77.3 & 1 \\
\hline CNG & 0.3 & 0.3 & 0.3 & 0.4 & 0.4 & 0.4 & 0.5 & 0.5 & 0.5 & 0.6 & 0.6 & 67.7 & 67.7 & 1 \\
\hline $\begin{array}{l}\text { Hydrogen } \\
\text { Jet Fuel (kerosene \& }\end{array}$ & 0.0 & 0.0 & 0.001 & 0.001 & 0.001 & 0.002 & 0.003 & 0.004 & 0.006 & 0.008 & 0.009 & 98.3 & 98.3 & 2.3 \\
\hline naphtha) & 26.5 & 25.9 & 26.0 & 26.1 & 26.5 & 26.9 & 27.3 & 27.8 & 28.6 & 29.2 & 29.9 & 86.4 & 86.4 & 1 \\
\hline Aviation Gasoline & 0.3 & 0.3 & 0.3 & 0.3 & 0.3 & 0.3 & 0.3 & 0.3 & 0.3 & 0.3 & 0.3 & 85.0 & 85.0 & 1 \\
\hline Total & 241.4 & 246.1 & 247.8 & 248.3 & 247.9 & 247.2 & 246.8 & 246.9 & 247.4 & 248.6 & 249.8 & & & \\
\hline \multicolumn{15}{|c|}{ Based on the GHG emission reduction targets specified in the EISA. } \\
\hline \multicolumn{15}{|c|}{$\begin{array}{l}\text { AFCI } 2010 \text { (baseline fuels include all transport fuels in 2010): } 94.3 \mathrm{gCO}_{2} \mathrm{e} / \mathrm{MJ} \\
\text { AFCI 2020: } 91.8 \mathrm{gCO}_{2} \mathrm{e} / \mathrm{MJ}\end{array}$} \\
\hline \multicolumn{15}{|c|}{ Total $\mathrm{CO}_{2} \mathrm{e}$ emissions in $2010=2727$ million tonnes } \\
\hline \multicolumn{15}{|c|}{ Total $\mathrm{CO}_{2} \mathrm{e}$ emissions in $2020=2747$ million tonnes } \\
\hline \multicolumn{15}{|c|}{2020 AFCI goal: 84.9 (10\% reduction from the baseline) } \\
\hline \multicolumn{15}{|c|}{$2020 \mathrm{CO}_{2} \mathrm{e}$ emissions if $10 \%$ AFCI goal is met $=2535$ million tonnes } \\
\hline LCFS $\mathrm{CO}_{2} \mathrm{e}$ emissi & n reduc & & 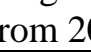 & חרי & & & $211 n$ & & & & & & & \\
\hline
\end{tabular}


Table S5. Estimates of fuel use and $\mathrm{CO}_{2} \mathrm{e}$ emissions if the biofuel target mandated in the Energy Independence and Security Act (EISA) is assumed to be fully met.

\begin{tabular}{|c|c|c|c|c|c|c|c|c|c|c|c|c|c|c|c|c|}
\hline \multirow[b]{2}{*}{ Fuel type } & \multicolumn{13}{|c|}{ Transportation fuel use (BGGE/yr) } & \multirow[b]{2}{*}{$\begin{array}{c}\text { CI } \\
(2010)\end{array}$} & \multirow[b]{2}{*}{$\begin{array}{c}\text { CI } \\
(2022)\end{array}$} & \multirow[b]{2}{*}{ EER } \\
\hline & 2010 & 2011 & 2012 & 2013 & 2014 & 2015 & 2016 & 2017 & 2018 & 2019 & 2020 & 2021 & 2022 & & & \\
\hline Gasoline & 142.9 & 146.1 & 145.0 & 143.2 & 140.9 & 138.0 & 135.6 & 133.3 & 131.0 & 129.6 & 127.8 & 125.3 & 123.5 & 95.9 & 95.9 & 1 \\
\hline Conventional biofuel & 8.0 & 8.4 & 8.9 & 9.3 & 9.7 & 10.1 & 10.1 & 10.1 & 10.1 & 10.1 & 10.1 & 10.1 & 10.1 & 99.4 & $76.7^{\#}$ & 1 \\
\hline Cellulosic biofuel & 0.1 & 0.2 & 0.3 & 0.7 & 1.2 & 2.0 & 2.9 & 3.7 & 4.7 & 5.7 & 7.1 & 9.1 & 10.8 & 38.4 & $38.4^{*}$ & 1 \\
\hline Diesel & 62.1 & 63.6 & 65.3 & 66.5 & 66.8 & 67.0 & 67.5 & 68.2 & 69.0 & 69.6 & 70.4 & 71.0 & 71.4 & 94.7 & 94.7 & 1 \\
\hline $\begin{array}{l}\text { Biomass based } \\
\text { biodiesel }\end{array}$ & 0.9 & 1.0 & 1.3 & 1.6 & 1.8 & 2.1 & 2.3 & 2.6 & 2.9 & 3.1 & 3.4 & 3.6 & 4.1 & 47.95 & $48.0^{+}$ & 1 \\
\hline Electricity & 0.2 & 0.2 & 0.2 & 0.2 & 0.2 & 0.2 & 0.2 & 0.2 & 0.3 & 0.3 & 0.3 & 0.3 & 0.3 & 164.4 & 164.4 & 3 \\
\hline LPG & 0.1 & 0.1 & 0.1 & 0.1 & 0.1 & 0.1 & 0.1 & 0.1 & 0.1 & 0.1 & 0.1 & 0.1 & 0.1 & 77.3 & 77.3 & 1 \\
\hline CNG & 0.3 & 0.3 & 0.3 & 0.4 & 0.4 & 0.4 & 0.5 & 0.5 & 0.5 & 0.6 & 0.6 & 0.6 & 0.6 & 67.7 & 67.7 & 1 \\
\hline Hydrogen & 0.0 & 0.0 & 0.0 & 0.0 & 0.0 & 0.0 & 0.0 & 0.0 & 0.0 & 0.0 & 0.0 & 0.0 & 0.0 & 98.3 & 98.3 & 2.3 \\
\hline $\begin{array}{l}\text { Jet Fuel (kerosene \& } \\
\text { naphtha) }\end{array}$ & 26.5 & 25.9 & 26.0 & 26.1 & 26.5 & 26.9 & 27.3 & 27.8 & 28.6 & 29.2 & 29.9 & 30.5 & 31.0 & 86.4 & 86.4 & 1 \\
\hline Aviation Gasoline & 0.3 & 0.3 & 0.3 & 0.3 & 0.3 & 0.3 & 0.3 & 0.3 & 0.3 & 0.3 & 0.3 & 0.3 & 0.3 & 85.0 & 85.0 & 1 \\
\hline Total & 241.4 & 246.1 & 247.8 & 248.3 & 247.9 & 247.2 & 246.8 & 246.9 & 247.4 & 248.6 & 249.8 & 250.9 & 252.2 & & & \\
\hline
\end{tabular}

AFCI 2010 (baseline fuels include all transport fuels in 2010): $94.3 \mathrm{gCO}_{2} \mathrm{e} / \mathrm{MJ}$

AFCI 2020: $91.4 \mathrm{gCO}_{2} \mathrm{e} / \mathrm{MJ}$ (3.1\% reduction from AFCI 2010)

AFCI 2022: $90.1 \mathrm{gCO}_{2} \mathrm{e} / \mathrm{MJ}$ (4.4\% reduction from AFCI 2010)

The carbon intensities of jet fuel and aviation gasoline are based on Table A-29 of EPA, US Inventory of US

Greenhouse Gas Emissions and Sinks: 1990-2006

(http://epa.gov/climatechange/emissions/downloads/08_CR.pdf) and adjusted for lifecycle emissions, assuming upstream emissions account for about 20\% of total GHG emissions from petroleum (Table S6).

Table S6. Estimates of lifecycle carbon intensities for jet fuel and aviation gasoline.

\begin{tabular}{lccc}
\hline & $\begin{array}{c}\text { Carbon content } \\
\left(\mathrm{gCO}_{2} \mathrm{e} / \mathrm{MJ}\right), \mathrm{HHV}\end{array}$ & $\begin{array}{c}\text { Carbon content } \\
\left(\mathrm{gCO}_{2} \mathrm{e} / \mathrm{MJ}\right), \mathrm{LHV}\end{array}$ & $\begin{array}{c}\text { Estimated lifecycle emissions } \\
\text { (gCO } \mathrm{e} / \mathrm{MJ}), \mathrm{LHV} \text { (assuming 20\% } \\
\text { emissions are from upstream) }\end{array}$ \\
\hline Aviation gasoline & 65.6 & 70.9 & 85.1 \\
Jet fuel (kerosene and naphtha) & 67.2 & 72.0 & 86.4 \\
\hline
\end{tabular}




\section{References}

S1. CARB. Staff Report: Proposed Regulation to Implement the Low Carbon Fuel Standard - Initial Statement of Reasons Volume 1: Staff Report; California Air Resources Board: 2009. Available from http://www.arb.ca.gov/fuels/lcfs/030409lcfs_isor_vol1.pdf.

S2. $\quad$ Parker, N.; Tittmann, P.; Hart, Q.; Lay, M.; Cunningham, J.; Jenkins, B. Strategic Assessment of Bioenergy Development in the West: Spatial Analysis and Supply Curve Development; 2008. Available from http://www.westgov.org/wga/initiatives/transfuels/Task\%203.pdf.

S3. Antares Group Inc. Strategic Assessment of Bioenergy Development in the West: Bioenergy Conversion Technology Characteristics; 2008. Available from http://www.westgov.org/wga/initiatives/transfuels/Task\%202.pdf.

S4. $\quad$ Nelson, R.; Skog, K.; Mallory, M. P.; Rummer, R.; Barbour, R. J. Strategic Assessment of Bioenergy Development in the West: Biomass Resource Assessment and Supply Analysis for the WGA Region; Kansas State University and the U.S. Forest Service 2008. Available from http://www.westgov.org/wga/initiatives/transfuels/Task\%201.pdf.

S5. U.S. DOE. VISION; Argonne National Laboratory, U.S. Department of Energy: 2008. Available from http://www.transportation.anl.gov/software/VISION/index.html.

S6. Melaina, M. VISION-CA model can be downloaded at http://www.lcfs.ucdavis.edu/vision.html: 2008.

S7. CARB. Comparison of Greenhouse Gas Reductions for the United States and Canada Under U.S. CAFE Standards and California Air Resources Board Greenhouse Gas Regulations; California Air Resources Board: Sacramento, CA, February, 2008.

S8. CARB. California Procedures for Evaluating Alternative Specifications for Phase 3 Reformulated Gasoline Using the California Predictive Model; California Air Resources Board: Sacramento, CA, 2008.

S9. General Motors Corporation. General Motors Corporation 2009-2014 Restructuring Plan. Presented to U.S. Department of the Treasury; February 17, 2009.

S10. Chrysler. Chrysler Restructuring Plan for Long-Term Viability; February 17, 2009.

S11. Ford Motor Company. Ford Motor Company Business Plan. Submitted to Senate Banking Committee; 2009.

S12. EIA. Annual Energy Outlook 2009; Energy Information Administration: Washington, DC, 2009. Available from www.eia.doe.gov/oiaf/aeo/.

S13. H. R. 6: Energy Independence and Security Act. In (P.L. 110-140, H.R. 6), 2007. 